\title{
Small-Scale Experimental Model of a Helical Steel Pile as the Heat Exchanger in Ground Source Heat Pumps
}

\author{
Leya R. Kober ${ }^{1}$, Sarah R. Nicholson ${ }^{1}$, Sylvie Antoun ${ }^{1}$, Seth B. Dworkin ${ }^{1 *}$ \\ ${ }^{1}$ Mechanical and Industrial Engineering, Ryerson University, Toronto, Canada \\ *seth.dworkin@ ryerson.ca
}

\begin{abstract}
Ground Source Heat Pump (GSHP) technologies are a way to obtain clean, renewable energy to heat and cool buildings by using the moderate year-round temperature of the ground as a heat-transfer medium. Across Canada, there is great potential to implement GSHPs due to soil conditions and an increasing demand for sustainable energy, especially in northern regions where conventional heating and cooling systems running on fuel are nearing their end-of-life. However, for these technologies to be more appealing in Canada, they need to be made more economically efficient, since high costs are a limiting factor in their implementation. As a solution, helical steel piles may be used in place of deep boreholes as the primary heat exchangers in the GSHP system, as well as for structural support for building foundations. With the dual functionality of helical steel piles, there is potential to save materials and reduce associated costs.

To explore the thermodynamic capabilities of helical steel piles in GSHPs, a small-scale model of a complete single helical steel pile with a custom internal pipe layout was created with the combination of 3D printing and custom machining. The model pile was placed in a tank of soil medium and put in an insulated jacket to simulate soil conditions of the ground and minimize external interference. With sensors to measure temperatures and fluid flow, a variety of tests were run on the small-scale model to imitate field operation. Both laminar and turbulent flows were used to measure the effects of flow conditions on heat exchange performance. Alongside observing the effects of changing flow rates, the temperature distribution in the soil medium was measured radially.

Currently, there is no existing experimental data for this pile setup, so this research is essential for influencing possible design, operation, and future improvements to the equipment. The temperature gradients measured in the soil will inform spacing of the piles in an array, and the flow results will help with performance optimization while minimizing energy use. Finally, this experiment will provide necessary validation for numerical models of the pile which will help characterize their functionality and inform design. The experiment provides a fundamental foundation for the use of foundational piles in GSHPs in Canada - and supports international research into this technology.
\end{abstract}

